\title{
BMJ Who is more likely to use doctor-rating Open websites, and why? A cross-sectional study in London
}

\author{
Matteo Maria Galizzi, ${ }^{1}$ Marisa Miraldo, ${ }^{2}$ Charitini Stavropoulou, ${ }^{3}$ Mihir Desai, ${ }^{4}$ \\ Wikum Jayatunga, ${ }^{4}$ Mitesh Joshi, ${ }^{4}$ Sunny Parikh ${ }^{5}$
}

To cite: Galizzi MM, Miraldo M, Stavropoulou C, et al. Who is more likely to use doctor-rating websites, and why? A cross-sectional study in London. BMJ Open 2012:2:e001493.

doi:10.1136/bmjopen-2012001493

- Prepublication history and additional material for this paper are available online. To view these files please visit the journal online (http://dx.doi.org/10.1136/ bmjopen-2012-001493).

Received 1 June 2012 Accepted 4 October 2012

This final article is available for use under the terms of the Creative Commons Attribution Non-Commercial 2.0 Licence; see http://bmjopen.bmj.com

For numbered affiliations see end of article.

Correspondence to Dr Marisa Miraldo; m.miraldo@imperial.ac.uk

\section{ABSTRACT}

Objectives: To explore the extent to which doctorrating websites are known and used among a sample of respondents from London. To understand the main predictors of what makes people willing to use doctorrating websites.

Design: A cross-sectional study.

Setting: The Borough of Hammersmith and Fulham, London, England.

Participants: 200 individuals from the borough.

Main outcome measures: The likelihood of being aware of doctor-rating websites and the intention to use doctor-rating websites.

Results: The use and awareness of doctor-rating websites are still quite limited. White British subjects, as well as respondents with higher income are less likely to use doctor-rating websites. Aspects of the doctor-patient relationship also play a key role in explaining intention to use the websites. The doctor has both a 'complementary' and 'substitute' role with respect to Internet information.

Conclusions: Online rating websites can play a major role in supporting patients' informed decisions on which healthcare providers to seek advice from, thus potentially fostering patients' choice in healthcare. Subjects who seek and provide feedback on doctorranking websites, though, are unlikely to be representative of the overall patients' pool. In particular, they tend to over-represent opinions from non-White British, medium-low-income patients who are not satisfied with their choice of the healthcare treatments and the level of information provided by their GP. Accounting for differences in the users' characteristics is important when interpreting results from doctorrating sites.

\section{INTRODUCTION}

In recent years, both the NHS Plan ${ }^{1}$ and the NHS Improvement Plan, ${ }^{2}$ set out the changes required for the English NHS to become more patient-focused. Greater patient involvement in the running of the NHS has gone hand-in-hand with the policymakers' drive to improve the quality of

\section{ARTICLE SUMMARY}

Article focus

- To explore the awareness of the existence of doctor-rating websites and their usage among a sample of respondents from London.

- To understand the main predictors of what makes people willing to use doctor-rating websites.

Key messages

- The share of actual users of doctor-rating websites is quite low.

- Subjects with White British background, as well as subjects with higher income are less likely to use doctor-rating websites.

- The doctor-patient relationship is a significant predictor of patients' intention to use doctorrating websites.

- The general practitioner (GP)-patient gender concordance is associated with higher intention to use the websites.

- Subjects who feel that their GP explains things clearly and is a valuable source of clear information, are less likely to use online rating websites.

- Subjects who feel that they are more satisfied with the level of choice of healthcare treatments are less likely to use online rating websites.

Strength and limitations of this study

- Our study contributes to the literature of online health information where evidence on the determinants of people's willingness to use doctorrating websites is limited.

- The main limitation of the study is that we use a convenience sample from one borough of London, UK and therefore results cannot be immediately generalised to the UK population.

public healthcare services. The 'bottom-up' approach to a more patient-centred NHS has typically focused on three main areas: (1) giving users more choice and personalisation; (2) making funding respond to users' choices and (3) engaging users through greater involvement. ${ }^{3}$ Lord Darzi's 2008 report 'High Quality Care For All-The 
Next Stage Review ${ }^{4}$ acknowledged that improvements to the NHS should focus on improving the quality of services, and that the best way of achieving this would be to ensure that services are locally responsive to the needs of the community, for instance, by empowering providers and patients as decentralised decision-makers in order to foster a culture of continuous quality improvement and innovation.

Websites, such as the NHS Choices and Dr Foster Intelligence, have been developed with the explicit aim of informing patients about the services that the NHS provides and therefore allowing a better choice of physicians and treatments. In principle, doctor-rating websites can have a profound impact on public involvement and patients' choice, as they enable patients to make more informed decisions on where to seek healthcare, and thus to engage more often in active choices concerning their health. In practice, however, relative little evidence is available on whether, and to what extent, doctor-rating websites are actually known and actively used in the UK.

A study by the Kings Fund ${ }^{5}$ explored the information sources used by patients in making decisions about where to receive care. Only $4 \%$ of the patients used the NHS Choices website, with the majority instead drawing information from their own experiences $(41 \%)$, and advice from their general practitioner (GP) $(36 \%)$. Similarly, a national survey on patients' choice by the Department of Health found that the NHS Choices website was only used by $5 \%$ of respondents. ${ }^{6}$ These figures are consistent with the evidence from the USA where usage of doctorrating websites is still quite low. ${ }^{7}$ Moreover, very little is known about the profile of individuals who are more likely to make active use of these sites. Appleby and Alvarez $^{9}$ found that women in England desire patient choice more than men (69-56\%), suggesting that women may also be more likely to use sources of information such as rating sites. This is in line with findings from the USA where women and younger adults are more active 'online health information seekers'. ${ }^{10}$

The aim of this study was to contribute to fill these gaps by providing more direct evidence on, first, the extent to which doctor-ratings websites are known and used among a sample of respondents in a borough of London; and, second, the most significant predictors of the fact that people are willing to use doctor-rating websites.

\section{METHODS}

We conducted a self-administered survey to assess the extent and the determinants of (1) the awareness of the existence of doctor-rating websites; (2) the level of actual usage of those websites; (3) the intention to use doctor-rating websites in the future.

\section{Questionnaire design}

Prior to the data collection, a pilot study was conducted. The aim of the pilot study was to gain an understanding of the practicalities associated with giving out questionnaires and collecting responses. After listening to feedback from pilot respondents, and looking at results from the pilot study, several changes were made to make the questionnaire easier to understand. The changes related to the content, phrasing and ordering of questions.

The content of the final questionnaire was based on findings from the preliminary literature review and was designed in a number of sections (see online supplementary appendix for full questionnaire). In particular, section A focuses on the awareness of online rating websites, while section $\mathrm{B}$ assesses actual usage of online rating websites. Section $\mathrm{C}$ measures the willingness to use the online rating websites in the future, and explores which aspects of the healthcare providers and which sources of information are perceived as being important factors in making decisions about where to receive healthcare. Section D assesses the individual contribution to online rating sites, while section $\mathrm{E}$ focuses on aspects of the doctor-patient relationship and attitudes and dimensions of patient choice. Finally section F controls for internet usage, while section $\mathrm{G}$ collects a broad range of sociodemographic characteristics.

Closed questions were used, worded in a manner easy to understand. A limited number of responses were provided, either with binary options (eg, yes or no), or with a numerical Likert scale ranging from 1 to 5, with a further option for 'Not sure'.

A list of variables with a brief description is discussed in the statistical analysis section and is summarised in the online supplementary table S1 in the appendix.

\section{Ethical approval, informed consent and confidentiality of responses}

We completed the checklist for research ethics approval from Imperial College London. As interviews were intended to be conducted in public places among respondents from the general population, the study involved no risk or harm of any type to respondents, no link with clinical data was expected to take place, and no incentives were going to be paid to respondents, the study fitted all the criteria in the first-stage checklist with no further formal application to the Imperial College Research Ethics Committee.

At the beginning of each interview, interviewers showed credentials as research assistants at the University of London, informed respondents that their answers were anonymous and would remain strictly confidential, and that all responses and data were going to be used for the purposes of scientific research only. Informed consent by respondents was then given at the beginning of each interview.

\section{Data collection}

The survey was conducted in the field by the researchers involved in the paper. The borough of Hammersmith and Fulham was chosen for the location of the field survey because it is a transport hub in Central West 
London, and hosts many offices and several major business centres. The four interviewers went to different public locations within the borough (underground stations, high street and residential areas) at different times during the day (early morning, midday and in the evening) and in different days of the week (including weekends). By covering different times and locations within the borough, we aimed at being able to approach both working and non-working members of the public. During the surveys in the field, the interviewers approached every third male and third female that would pass by them.

Sample size calculations were based on the intended objective to look at the correlation coefficient between the likelihood of using the websites on the one hand, and a typical survey response, on the other. The minimum sample size to test the null hypothesis of no significant correlation between these two variables was calculated given the most conservative assumption that the correlation coefficient between the variables in the population was in the region of 0.2 (a 'low' effect size, the variance of one variable accounting for just $4 \%$ of the variance of the other). Under the assumptions that all variables are normally distributed, a bidirectional test (both positive and negative correlation were expected) with $95 \%$ significance level reaches a standard $80 \%$ power level at a minimum sample of $n=200$ subjects. ${ }^{11}$ We thus targeted a sample size of 200 respondents. The envisaged target was then readily achieved, since only 68 subjects who were initially approached refused to take part to the survey, with a final response rate of $74 \%$.

\section{Statistical analysis}

Besides a correlation analysis, we carried out a multiple regression analysis which aims to explore the determinants of (1) being either aware or not of doctor-rating websites and (2) the individual intention of using these websites in the future.

The dependent variable in the first case is modelled as a binary variable (Awareness) taking values 1 or 0 for the respondents who reported to be aware or unaware of the websites, respectively. The second dependent variable is instead modelled as a discrete ordered variable (IntentionToUse) taking values 1,2 and 3 for subjects reporting to be 'not likely', 'quite likely' and 'likely' to use the websites in the future, respectively.

The explanatory variables $\left(\mathrm{X}_{\mathrm{i}}\right)$ include the variables described in the online supplementary table S1, namely: individual sociodemographic characteristics; a set of variables on the characteristics of the healthcare providers that the respondents consider important for making their decisions on where to receive healthcare; a set of variables on the sources of information that are important in making decisions about where to receive healthcare; two dummy variables that capture whether the patient's gender and age are the same with, or within a comparable range, respectively, than the gender and age of her GP; a set of variables that describe the respondents' feelings about their relationship with their doctor; a variable indicating the level of participation of the respondents in their GPs' decisions; a set of variables on patients' satisfaction with the level of choice in their healthcare decisions; a dummy variable controlling for whether the subjects had Internet access at home or at work; a variable on awareness of the existence of doctorrating websites; and a variable on whether the subject always asks to see the same GP (see supplementary table S1 for variables' details). The choice of the explanatory variables was further informed by the bivariate correlation analysis reported in the online supplementary table S2 in the appendix.

We used a binary logistic and an ordered logistic model to fit the Awareness and the IntentionToUse discrete variables, respectively, to ensure a reasonable comparability between the empirical results obtained for the two set of regressions. The two models, in fact, only differ in the number of values that the dependent variables can take, while the underlying structure of the error terms follows the same standardised logistic distribution. The logistic specification is particularly appealing because its results can be readily expressed in terms of OR. We have, however, conducted a robustness check by replicating the multiple regression analysis using the alternative binary and ordered probit specifications. The two sets of regressions provide consistent estimates and results that are qualitatively fully aligned. Results of the probit specifications are available, on request, from the authors.

All the regression analysis has been conducted using STATA V.11.

\section{RESULTS}

\section{Sample and descriptive statistics}

Descriptive statistics of all the dependent and independent variables for the resulting sample of respondents to our survey are provided in detail in the online supplementary table S1, and here we briefly report their main aspects.

Our sample consisted of 200 subjects. Comparing it with the Census data for the borough the mean age of our sample was slightly older than that for the borough (39.57 years compared to 35.2 years). ${ }^{12}$ Our sample, however, was closer to the national mean age of 38.5 years. The range of ages seems to show a positive skew, with a greater frequency of people aged 40 years and under. This is consistent with the 2001 census data for Hammersmith and Fulham which showed that the borough contained a larger proportion of young people aged 20-29 (23.8\%) than the rest of England (12.66\%). ${ }^{12}$

Also, the sample had a slightly greater proportion of females than the borough (54.44-52\% respectively), and a lower proportion of 'White British' ethnicity (48.79\% compared to $58 \%$ for the borough). ${ }^{12}$ This is also significantly lower than figures for England, where White British account for $87 \%$ of the population. ${ }^{12}$ The sample 
contained $28.99 \%$ non-White respondents. This is higher than the 2001 census data for Hammersmith and Fulham which was $22 \%$ and significantly higher than the figures for England, showing non-White ethnic groups accounting for $9 \%$ of the total population. ${ }^{12}$ Our sample, therefore, allows controlling for high heterogeneity in ethnic background even with a limited sample size.

Regarding working status, 141 individuals were workers (10 of which reported to be currently unemployed), 33 students, 9 officially unemployed and 6 retired. Eleven respondents did not report their working status. The proportion of subjects who were not currently working, as given by the sum of the respondents who reported to be unemployed, retired or students, indeed amounts to $29 \%$ of the sample. The majority of actively working respondents reported an income within the £15-35000 bracket.

Our sample had a high percentage of people with higher-level qualifications: $46.24 \%$ of the sample had a university degree and $27.96 \%$ had a postgraduate degree. This is reflective of Hammersmith and Fulham, where $45 \%$ of the population have a qualification of degree level or higher, a figure which is significantly higher than in England, where only $19.8 \%$ have a degree or higher qualification. ${ }^{12}$

\section{Results on awareness}

Only 29 of our respondents were aware of the doctorrating websites they were asked about and only 6 reported to have used them.

In the online supplementary table S2 we present the set of bivariate correlations between the fact of being aware of the websites and each of the variables collected in the survey. As it can be seen, there is a positive correlation between having Internet access, or being aware of the NHS Choose and Book system, and being aware of the doctor-rating websites. Age exhibits a negative correlation, whereas the gender concordance with the GP, shows a positive correlation. Positive correlations with the awareness of doctor-rating websites also hold for respondents who think that those websites are important sources of information, or who see accessibility and financial performances of hospitals as important factors in making decisions regarding where to seek healthcare.

In table 1 we present the estimate results of four different specifications of the binary logistic regression for the dependent variable Awareness with different sets of regressors, which are presented in terms of the OR, together with the standard errors, and levels of significance.

Among the demographic factors, age and ethnicity are the only significant variables. Older individuals are less likely to be aware of the rating websites, which does not constitute a surprise, as they are usually less familiar with the use of internet in general. Moreover, in most specifications, White British and White non-British respondents appear less likely to be aware of the websites.
Among the broader sociodemographic factors, only income is sometimes (marginally) significant, pointing to the fact that respondents with higher reported levels of income tend to be less aware of the websites, while neither education or gender turn out to be significant predictors of awareness.

Looking at the characteristics of the providers that respondents consider important in making their decisions on where to receive healthcare, in one specification the reputation of the doctor has a strong positive effect, while both clinical and financial performance rates of the providers show negative significant effects. Thus, the respondents who consider the reputation of the doctor important in deciding where to receive care are more likely to be aware of the rating websites, while this is less often the case for respondents putting a higher weight on financial or clinical performance ratings.

Concerning the sources of information, respondents who consider the hospital statistics important in deciding where to receive care, turn out to be more likely of being aware of the rating websites, with an effect which is particularly significant and quite remarkable in terms of OR.

Furthermore, although in one specification the respondents who feel that their GPs spend sufficient time in their consultation are less likely to be aware of the internet rating websites, neither the statistical significance nor the estimated OR appear robust across specifications. Although all other variables on doctor-patient relationship were not significant, whenever included among the regressors, the gender match between the GP and the patient predicts higher awareness of the website ratings, with a noticeable effect as evident by the reported value of the OR.

As aforementioned, from those that were aware of the existence of doctor-rating websites only six have reported to have used these websites. In light of this low usage rate, and of the consequent limitations of conducting statistical estimations with very little variation in the dependent outcomes, we have thus focused the rest of the analysis on the determinants of the intention to use, rather than actual usage of, doctor-rating websites.

\section{Results on the likelihood to use online rating websites}

In the online supplementary table S2 we present the set of bivariate correlations between the intention to use the doctor-rating websites and each of the variables collected in the survey. As it can be noticed, there is a positive correlation between having internet access, and being aware of the doctor-rating websites. Both the age and the gender concordance with the GP show a positive correlation with the intention to use. Positive correlations with the willingness to use doctor-rating websites also hold for respondents who think that those websites, or hospital statistics, are important sources of information. Also the fact that respondents believe that online rating is a reliable measure is clearly correlated with the 
Table 1 ORs for the Binary Logit explaining the awareness of doctor-rating websites

\begin{tabular}{|c|c|c|c|c|}
\hline & Model 1 & Model 2 & Model 3 & Model 4 \\
\hline \multicolumn{5}{|l|}{ Awareness } \\
\hline Age & $0.953(0.0239)^{\star}$ & $0.931(0.0307)^{\star \star}$ & & \\
\hline Gender & $1.347(0.648)$ & $1.819(1.092)$ & & \\
\hline WhiteBritish & $0.595(0.309)$ & $0.841(0.524)$ & $0.401(0.276)$ & $0.0150(0.0292)^{\star \star}$ \\
\hline White Non British & $0.273(0.198)^{\star}$ & $0.398(0.324)$ & $0.228(0.200)^{\star}$ & $0.00399(0.00957)^{\star \star}$ \\
\hline Education & $1.105(0.341)$ & $1.396(0.534)$ & $1.279(0.438)$ & $1.682(1.399)$ \\
\hline Income & $0.952(0.157)$ & $0.943(0.169)$ & $0.708(0.132)^{*}$ & $0.228(0.180)^{*}$ \\
\hline HC_HospComp & & $1.173(0.366)$ & $1.353(0.442)$ & $2.237(1.825)$ \\
\hline HC_Clinical_Performance & & $0.691(0.245)$ & 0.527 (0.207) & $0.0342(0.0609)^{*}$ \\
\hline HC_Familiarity & & $0.710(0.170)$ & $0.756(0.202)$ & $2.564(2.096)$ \\
\hline HC_GP_Reputation & & 1.409 (0.509) & $1.611(0.599)$ & $13.57(19.95)^{*}$ \\
\hline HC_FinPerform & & $0.921(0.264)$ & $0.963(0.297)$ & $0.0783(0.0919)^{\star *}$ \\
\hline HC_Access & & $1.112(0.236)$ & $1.088(0.242)$ & $0.917(0.444)$ \\
\hline SI_GP_Advice & & $1.173(0.350)$ & $0.922(0.290)$ & $1.115(0.718)$ \\
\hline SI_HospStat & & $1.291(0.410)$ & $1.390(0.477)$ & $49.75(87.28)^{\star \star}$ \\
\hline SI_Family & & $0.935(0.361)$ & $0.614(0.273)$ & $0.146(0.186)$ \\
\hline SI_PastExp & & $0.762(0.275)$ & $1.202(0.499)$ & $0.284(0.343)$ \\
\hline SI_DoctorRating & & $0.938(0.261)$ & $0.933(0.271)$ & 1.859 (1.119) \\
\hline DOC_Listens & & & $0.416(0.324)$ & $1.182(2.244)$ \\
\hline DOC_Time & & & $1.289(0.950)$ & $0.00185(0.00580)^{\star \star}$ \\
\hline DOC_Explains & & & 2.533 (1.799) & $0.885(1.658)$ \\
\hline DOC_Friend & & & $0.752(0.535)$ & 15.62 (30.63) \\
\hline DOC_Trust & & & $0.930(0.583)$ & $3.173(4.555)$ \\
\hline Participation & & & $1.080(0.298)$ & 3.346 (2.835) \\
\hline Age Match & & & 2.247 (1.429) & $269.4(791.0)^{\star}$ \\
\hline Gender Match & & & $3.153(1.867)^{\star}$ & $32.77(61.36)^{*}$ \\
\hline SAT_C_GP & & & & $3.020(2.948)$ \\
\hline SAT_C_Hosp & & & & $0.802(1.134)$ \\
\hline SAT_C_Doc & & & & $2.794(3.411)$ \\
\hline SAT_C_Treatment & & & & $1.818(2.311)$ \\
\hline SAT_C_Time & & & & $0.735(0.550)$ \\
\hline Same GP & & & & $0.641(0.766)$ \\
\hline
\end{tabular}

intention to use them. Finally, positive correlations also hold for respondents who feel that their doctor has time to dedicate to them, or who see several aspects of healthcare providers-such as reputation, clinical and financial performances, waiting lists, accessibility-as important factors when making decisions on where to seek healthcare.

In table 2 we present the estimate results of six different specifications of the ordered logistic regression for the dependent variable IntentionToUse with different sets of regressors, which are presented in terms of the OR, together with the standard errors, and levels of significance.

Concerning sociodemographic variables, it turns out that White British, as well as respondents who reported income in higher brackets, said that they were less likely to use doctor-rating websites. Moreover, we do not find any effect of education, age and gender of the respondents on the likelihood of their intention to use (the results of the specifications including the age and gender variables are not reported in the table for the sake of space but are available from the authors upon request).

Looking at the characteristics of the healthcare providers that respondents perceived as important while making decisions on where to receive healthcare, our data suggest that those who consider clinical performance and doctor reputation (in most specifications) as important factors, are more likely to use doctor-rating websites. These results are consistent with the nature of the information provided in these websites. Also, and quite intuitively, subjects who consider the familiarity with their doctor an important factor to decide where to seek healthcare, tend to be less likely to intend to use websites.

Concerning the role of the different sources of information on the decisions of where to seek healthcare, respondents who see published hospital statistics as important sources of information are more likely to use the rating websites. On the other hand, those for whom GP advice is an important source of information for decision making are less likely to use doctor-rating websites. 
Table 2 ORs for the Ordered Logit explaining the likelihood to use doctor rating websites

\begin{tabular}{|c|c|c|c|c|c|c|}
\hline & m1 & m2 & m3 & m4 & m5 & m6 \\
\hline AgeMatch & $1.974(2.377)$ & $2.561(2.953)$ & $2.000(1.965)$ & $2.782(2.613)$ & $1.051(0.818)$ & $0.946(0.729)$ \\
\hline GenderMatch & $18.42(30.24)^{*}$ & $12.03(17.75)^{*}$ & $10.45(12.33)^{\star *}$ & $10.39(10.54)^{\star *}$ & $16.67(15.48)^{\star * \star}$ & $14.83(13.17)^{\star * \star}$ \\
\hline Awareness & $0.0531(0.108)$ & $0.0505(0.0971)$ & $0.0964(0.149)$ & $0.0758(0.0985)^{\star *}$ & $0.159(0.176)^{\star}$ & $0.147(0.152)^{*}$ \\
\hline HC_Clinical_Performance & $9.289(11.84)^{*}$ & $7.659(8.241)^{*}$ & $5.560(4.759)^{\star \star}$ & $3.401^{*}(2.253)$ & $4.395(2.653)^{\star \star}$ & $4.985(2.734)^{\star \star \star}$ \\
\hline HC_Familiarity & $0.359(0.287)$ & $0.468(0.282)$ & $0.371(0.220)^{\star}$ & $0.414^{*}(0.206)$ & $0.355(0.147)^{\star \star}$ & $0.351(0.141)^{\star \star \star}$ \\
\hline HC_GP_Reputation & $2.328(1.980)$ & 2.827 (2.106) & $3.608(2.542)^{*}$ & $4.410(2.753)^{\star *}$ & $2.903^{\star *}(1.374)$ & $2.776^{\star \star}(1.260)$ \\
\hline SI_GP_Advice & $0.170(0.173)^{\star}$ & $0.223(0.206)$ & $0.238(0.167)^{\star *}$ & $0.283(0.176)^{\star \star}$ & $0.344^{* *}(0.186)$ & $0.396^{*}(0.193)$ \\
\hline SI_HospStat & $14.26(18.84)^{\star *}$ & $13.74(15.60)^{* *}$ & $7.220(5.008)^{\star \star \star}$ & $6.550(4.200)^{\star \star \star}$ & $5.371^{\star * *}(2.932)$ & $5.133^{\star * *}(2.703)$ \\
\hline SI_DoctorRating & $1.596(1.636)$ & $1.067(0.958)$ & $1.424(0.851)$ & $1.461(0.770)$ & $2.245(0.835)^{\star \star}$ & $2.312^{\star *}(0.876)$ \\
\hline Reliable & 6.181 (7.691) & $8.682(9.969)^{*}$ & $6.492(4.993)^{\star *}$ & $7.586(5.561)^{\star * \star}$ & $4.457(2.351)^{\star * *}$ & $4.061^{* * *}(2.003)$ \\
\hline DOC_Listens & $141.9(424.8)^{\star}$ & $51.44(126.4)$ & $44.20(90.99)^{*}$ & $27.05(41.26)^{\star \star}$ & $22.03(28.29)^{\star \star}$ & $22.98(28.34)^{\star *}$ \\
\hline DOC_Explains & $0.00690(0.0183)^{*}$ & $0.00680(0.0148)^{\star *}$ & $0.00509(0.0105)^{\star \star}$ & $0.00695(0.0124)^{\star \star \star}$ & $0.0120(0.0171)^{\star \star \star}$ & $0.0124(0.0169)^{\star \star \star}$ \\
\hline DOC_Friend & $12.88(29.23)$ & $8.375(14.65)$ & $16.48(22.41)^{\star *}$ & $19.66(22.45)^{\star \star \star}$ & $8.718(8.047)^{\star \star}$ & $7.781(6.896)^{\star \star}$ \\
\hline Participation & $5.473(5.255)^{\star}$ & $5.818(5.410)^{\star}$ & $5.171(3.664)^{\star \star}$ & $4.162(2.687)^{\star \star}$ & $2.349(1.126)^{\star}$ & $2.228(1.036)^{\star}$ \\
\hline SAT_C_GP & $17.03(27.58)^{\star}$ & $8.038(10.23)$ & $6.593(6.659)^{\star}$ & $5.410(4.048)^{\star \star}$ & $4.692(2.889)^{\star \star}$ & $4.377(2.484)^{\star \star \star}$ \\
\hline SAT_C_Hosp & $21.93^{\star \star}(33.71)$ & $22.86(30.90)^{\star \star}$ & $30.01(33.63)^{\star \star \star}$ & $34.38(35.43)^{\star \star \star}$ & $17.95(15.52)^{\star \star \star}$ & $11.11(7.578)^{\star \star \star}$ \\
\hline SAT_C_Treatment & $0.0515(0.0764)^{\star \star}$ & $0.0561(0.0794)^{\star \star}$ & $0.111(0.106)^{\star \star}$ & $0.147(0.125)^{\star \star}$ & $0.145(0.111)^{\star \star}$ & $0.111(0.0788)^{\star \star \star}$ \\
\hline WhiteBritish & $0.0137(0.0318)^{*}$ & $0.0409(0.0738)^{\star}$ & $0.0542(0.0782)^{\star \star}$ & $0.0539(0.0690)^{\star \star}$ & $0.0909(0.0890)^{\star \star}$ & $0.105(0.0973)^{\star \star}$ \\
\hline Income & $0.416(0.190)^{\star}$ & $0.382(0.162)^{\star \star}$ & $0.449(0.154)^{\star \star}$ & $0.513(0.154)^{\star \star}$ & $0.476(0.129)^{\star * \star}$ & $0.462(0.120)^{\star \star \star}$ \\
\hline SAT_C_Doc & $0.242(0.468)$ & $0.243(0.374)$ & $0.148(0.161)^{*}$ & $0.135(0.144)^{\star}$ & $0.427(0.321)$ & \\
\hline SI_PastExp & $0.670(0.787)$ & $0.590(0.650)$ & $0.535(0.576)$ & $0.551(0.250)$ & & \\
\hline Education & $0.486(0.526)$ & $0.583(0.554)$ & $0.683(0.443)$ & $0.610(0.328)$ & & \\
\hline HC_Access & 1.046 (0.659) & $1.124(0.678)$ & $1.241(0.564)$ & $1.347(0.565)$ & & \\
\hline HC_PastExp & $1.030(0.578)$ & $0.914(0.487)$ & $0.930(0.397)$ & & & \\
\hline SI_Family & 1.208 (1.357) & $1.305(1.484)$ & $1.439(1.458)$ & & & \\
\hline DŌC_Time & $1.223(2.118)$ & 2.099 (3.261) & 2.594 (3.547) & & & \\
\hline DOC_Trust & $0.153(0.327)$ & 0.608 (0.983) & 0.460 (0.629) & & & \\
\hline WEB_Access & $1.122(4.345)$ & $0.558(1.763)$ & $0.483(0.918)$ & & & \\
\hline HC_Waiting & $0.960(0.806)$ & $1.097(0.846)$ & & & & \\
\hline HC_HospComp & $1.200(0.929)$ & $0.790(0.540)$ & & & & \\
\hline HC_CloseHome & $0.930(0.726)$ & $0.790(0.516)$ & & & & \\
\hline HC_FinPerform & $0.610(0.621)$ & $0.692(0.588)$ & & & & \\
\hline SAT_CC_Time & $1.449(1.441)$ & $1.530(1.280)$ & & & & \\
\hline WhiteNonBritish & $0.742(1.790)$ & $0.493(1.041)$ & & & & \\
\hline CB_AWARE & $1.422(3.158)$ & & & & & \\
\hline CB_Use & $83.93(354.7)$ & & & & & \\
\hline _cut1 & $\begin{array}{l}9454769.9 \\
(63313549.3)^{\star *}\end{array}$ & $\begin{array}{l}2474784.8 \\
(15197453.2)^{\star \star}\end{array}$ & $\begin{array}{l}3131224.6 \\
(18256829.6)^{\star *}\end{array}$ & $\begin{array}{l}2460471.3 \\
(13260544.4)^{\star \star *}\end{array}$ & $\begin{array}{l}10470831.2 \\
(45550085.5)^{\star \star \star}\end{array}$ & $\begin{array}{l}13892352.4 \\
(59299449.7)^{\star \star \star}\end{array}$ \\
\hline _cut2 & $\begin{array}{l}7.05660 e+09 \\
(5.66892 e+10)^{\star * \star}\end{array}$ & $\begin{array}{l}1.22556 \mathrm{e}+09 \\
(8.86204 \mathrm{e}+09)^{\star \star \star}\end{array}$ & $\begin{array}{l}1.14387 \mathrm{e}+09 \\
(7.69789 \mathrm{e}+09)^{\star \star \star}\end{array}$ & $\begin{array}{l}674102348.3 \\
(4.20283 e+09)^{\star \star \star}\end{array}$ & $\begin{array}{l}1.42570 e+09 \\
(7.17551 e+09)^{\star \star \star}\end{array}$ & $\begin{array}{l}1.60379 \mathrm{e}+09 \\
(7.78799 \mathrm{e}+09)^{\star \star \star}\end{array}$ \\
\hline
\end{tabular}

Exponentiated coefficients; SE in parentheses.

${ }^{*} \mathrm{p}<.10,{ }^{* *} \mathrm{p}<.05,{ }^{* * *} \mathrm{p}<.01$. 
Also the nature of the doctor-patient relationship seems to play a key role in explaining whether respondents intend to use online rating websites. First, patients with GPs of the same gender tend to be more likely to use the websites. Second, respondents for whom the doctor is able to listen to them, and who perceive the nature of the relationship with their GP as friendly, also tend to be more likely to use the websites. Third, respondents who feel that their doctor explains things clearly are less likely to use online rating websites. Fourth, it also transpires that the more autonomy patients have in their healthcare decisions, the more likely they are to be willing to use the rating websites.

Finally, concerning, the interaction between levels of satisfaction with healthcare services within the NHS, and the intention to use doctor-rating websites, note that those that have reported to be more satisfied with the level of choice of GP, and with the amount of choice of the hospital to receive outpatient appointments, are more likely to use these websites. On the other hand, the respondents that are more satisfied with the level of choice of treatments are less likely to use the websites.

\section{DISCUSSION}

In this section we briefly discuss our main findings on the determinants of the intention to use doctor-rating websites and the level of awareness and actual usage of these websites. Results show that sociodemographic characteristics (in particular income and ethnicity) and the doctor-patient relationship are significant determinants of the intention to use these websites. Regarding the latter we further show that, from a patient's perspective, the doctor and the Internet can be seen as being both 'complementary' and 'substitute' sources of information. Yet, we find that awareness and usage of doctor-rating websites is low in our sample. In what follows we will discuss these results and relate them to evidence from the literature.

\section{Intention to use}

On the sociodemographic variables the results that show that White British and respondents who reported income in higher brackets said that they were less likely to use doctor-rating websites, are partly in contrast to what found by the previous literature ${ }^{13-15}$ and can signal that White British subjects and respondents with higher self-reported income may feel less in need of checking online doctor ratings, perhaps because they may also have private, or employer-paid, health insurance schemes, or because they are in the position of directly accessing alternative sources of information through their networks of acquaintances. Another possible explanation may be that White British individuals may trust less information that exists online and they have more concerns about confidentiality issues as shown in a study among different socioeconomic groups in the US by Brodie et al. ${ }^{16}$ As the estimated effect of these variables appear to be robust across all empirical specifications, these findings seem to suggest that online doctor-rating websites are likely to be particularly attractive to subjects with non-White British ethnicity and less favoured economic background.

On the other hand, the lack of statistical significance in the ordered logit estimates, seems to suggest that, while age can be a significant factor in explaining the awareness of Internet for health information, it is not significantly explaining the intention to use doctorrating websites once subjects are made aware of their existence. The analogous lack of significance for the respondents' gender, on the other hand, does not support the view that women in the UK may be more likely to use patients' sources of information and rating websites, although they have been found to desire patient choice more than men. ${ }^{9}$ Both results differ from the findings from the literature on the use of online information. The literature has shown that sociodemographic characteristics are major determinants of usage of online health information. In particular, women and younger adults are more active 'online health information seekers'. ${ }^{10}{ }^{17-20}$ Education has also been found to determine the usage of online and offline health information. Cotton and Gupta ${ }^{15}$ and Diaz et al, ${ }^{13}$ carried out research into the characteristics of online and offline health information seekers and found that less educated individuals were less likely to be users of online health information.

Therefore even though, according to our findings, intentions to use do not differ significantly across all sociodemographic characteristics, actual usage may be greatly determined by access rather than only by intention to use, with the former substantially differing according to socioeconomic and sociodemographic characteristics. That is, there may exist income-related, education-related or age-related barriers to actual access that prevent individuals from using doctor-rating sites even though their intentions to use them are similar.

From the perspective of the doctor-patient relationship, the finding that patients with GPs of the same gender tend to be more likely to use the websites is of particular interest, and it is consistent with the analogous effect found for the likelihood of being aware of those websites. Considered together these findings point to the possible explanation that the doctor and the Internet may sometimes be seen by patients as 'complementary', rather than alternative, information channels. ${ }^{14}$ This interpretation is further confirmed by the finding that respondents for whom the doctor is able to listen to them, and who perceive the nature of the relationship with their GP as friendly, also tend to be more likely to use the websites.

The doctor-patient gender concordance, in fact, has often been reported in the literature as a factor associated with higher patient satisfaction with the consultation as well as better outcomes. ${ }^{21}$ If we interpret the gender match variable as an indication of satisfaction 
with the consultation, our finding indicates that the intention to use (as well as being aware of) the doctorrating websites is not necessarily the result of a poor consultation. Instead, the Internet and the doctor are likely to be seen as complementary, rather than alternative, information channels. This could explain why patients that consider hospital statistics as a good source of information are more likely to use these websites. Indeed, this type of information might not be provided by the doctor in a consultation.

Indeed, a study by Stevenson et $a l^{22}$ shows that although patients use the Internet increasingly more, they show no intention of doing so with the aim of disrupting the existing balance of roles during the doctorpatient consultation. They all mentioned the Internet as an additional resource of health and healthcare information. Other evidence suggests that patients with hypertension who search for more information on the Internet, in addition to that they receive from their doctor, may be more engaged in their treatment, and therefore more willing to adhere to medication prescribed by them. ${ }^{23}$

Finally, the positive association between willingness to use doctor-rating websites and levels of satisfaction with the level of choice of GP, and of outpatient appointments in the hospital, can be considered as reinforcing the above-discussed interpretation that some dimensions of the doctor-patient relationship may be 'complementary' with online information. For instance, patients who are more satisfied with their GP because they feel the latter is more friendly and empathic may also be more likely to engage more actively with health and healthcare information more generally. These results, together with the finding that the respondents who are more satisfied with the level of choice of treatments are less likely to use the websites, suggest that the choice of doctors and providers may be seen as only instrumental for the choice of treatment, and therefore respondents that are happy with treatment choice levels are less likely to shop around for different doctors' opinions.

On the other hand, there may be other dimensions in the patient-doctor relationship which seem to rather point to a 'substitute' relationship with information on the Internet. For instance, the fact that respondents who feel that their doctor explains things clearly and consider the advice given by the GP as being important are less likely to use online rating websites, suggests that when they are generally more satisfied with the feedback provided by their doctor they are less concerned about finding about alternative doctors and compare them with their current GP.

This result on a 'substitute relationship' is consistent with previous evidence by Diaz $e t a l^{13}$ that found that $11 \%$ of their respondents said they would rather use the Internet 'instead of seeing or speaking with their doctors', and that $59 \%$ of respondents 'did not discuss information with their doctors'. It also seems in line with the study by McMullan ${ }^{14}$ that indicates that patients who become dissatisfied with the information provided to them by the health professionals are more likely to seek confirmation of the information given as well as additional information on the Internet.

Our findings that online information can be used not only as 'substitute' but also, and perhaps mainly, as 'complementary' to several dimensions of the doctorpatient relationship do not seem to entail any particular evidence suggesting that online ratings may put in danger the doctor-patient relationship, an important aspect which has been raised in the literature. ${ }^{24} 25$ The 'complementarity' findings, in particular, seem consistent with the evidence from the USA which shows that the vast majority of the reviews by patients are generally rather positive. ${ }^{7} 826$ Taken together, this evidence can be seen as providing little support to the related concern that the likeliest to use online ratings and enter actual comments may be the most disgruntled patients. $^{27}$

As for the other aspects of the patient-doctor relationship, the finding that the more autonomous patients are in their healthcare decisions, the more willing they are to use the rating websites is also consistent with previous evidence: a study by McMullan, ${ }^{14}$ for instance, reports that patients would seek health information before a consultation 'to manage their own healthcare independently'. These may be the type of people who are 'more likely to be health-oriented' or 'health conscious', and therefore be more proactive in consultations. ${ }^{28}$

\section{Awareness and actual usage}

Only $15 \%$ of our sample were aware of the existence of these websites, indicating that the awareness and, consequently, usage of these online sources is still quite limited in the UK, although significantly higher than what the previous studies have shown. ${ }^{5}$

Concerning the low reported rate of active usage of doctor-rating websites, the finding is not too surprising given that the survey was done among a sample of respondents from the general population: the reason why many more respondents were aware of the online ratings than did actually use it may simply be because those subjects did not actually need to see a doctor.

These figures are substantially in line with previous evidence brought forward from the literature from the UK. $^{5}{ }^{6}$ A slow uptake of online ratings has also been reported in the US. It is indicative that only $6 \%$ of Americans were aware of Hospital Compare, the quality reporting website maintained by the Centres for Medicare and Medicaid Services (CMS). ${ }^{29}$ Gao et $a l^{8}$ analysed 386000 national ratings from 2005 to 2010 in the USA and showed that only one of six physicians among those included in the study had received some rating. Lagu et $a l^{7}$ also reported a low average number of ratings per physician.

The fact that even in the USA, a more market-oriented health system, the use of similar sites is not much higher may suggest that the slow uptake in the UK cannot be 
attributed only to the early stage of the 'choice' model. Considered together, these results may pose concerns on the reasons and consequences of the lack of patient awareness and usage of online health-related information.

Previous studies in the USA have reported a number of reasons behind this slow uptake, including (1) the preference for more traditional information channels, such as recommendations by family and friends; (2) the lack of time and (3) in many cases the fact that people do not recognise that the quality of care may vary. ${ }^{25}$

As for the UK, our study confirms that not only awareness of rating websites is still limited among a sample of respondents from the general public in London, but awareness and willingness to use per se do not seem a sufficient condition to guarantee active usage. This poses a double challenge from a clinician and health policy perspective. In fact, on the one hand, the documented correlation between online ratings and other measures of healthcare quality, including survey-based ratings and clinical quality indicators, ${ }^{30} 31$ necessarily requires that patients have already gone through three preliminary hurdles, namely (1) being aware of, (2) having effective access to and (3) being active users of the doctor-rating websites. If the ultimate goal is indeed the continuous enhancement of healthcare quality, the effective removal of this triple hurdle is likely to become the next priority to guarantee the full spread of online rating website.

On the other hand, while appropriate online and offline informational campaigns are likely to overcome the first hurdle, thus effectively raising patients' awareness of online ratings as a potential source of information on provider quality, informational campaigns alone can fail to grant effective access and trigger actual changes in behaviour. Alike in several other health contexts, in particular, 'nudging' behaviour may be difficult as a mere consequence of accessing more information.

If this is the case, other avenues should be explored to increase the active usage of rating websites by patients who are already aware of them. For instance, the evidence brought forward by the present study confirms the importance of the doctor-patient relationship as a factor determining individuals' awareness of and willingness to use online ratings ${ }^{25} 32-35$ and suggests that tailored behavioural interventions based on the doctor-patient relationship have the potential to help patients to overcome the last hurdle and actively engage with online ratings.

\section{Limitations of the study}

The convenience field survey was considered the most appropriate administration mode to involve a sample of respondents from the general population. An online survey, in fact, by exclusively reaching the segment of active internet users, would have failed to address the main goal of the study.

However, while dictated by practical issues, the convenience sampling is a limitation of the study, and tends to over-represent respondents who are currently not employed, such as unemployed, retired and students. Also the fact that the study was conducted in only one borough of London limits the possibility to immediately generalise the findings to the broader UK population.

In an attempt to make such limitations of smaller concern to enhance the external validity and generalisability of the analysis, we have (1) chosen a borough which comprises a mix of both affluent and deprived neighbourhoods from heterogeneous ethnic backgrounds; (2) conducted surveys in the field at different public locations and at different times of the day and of the week to approach both working and non-working members of the public and (3) controlled for a wide range of sociodemographic measures in the statistical analysis.

\section{CONCLUSIONS}

By collecting a broad range of information on the sociodemographic characteristics of the respondents, their views and perceptions of the most important aspects of healthcare quality, patient choice and doctor-patient relationship, the study explicitly explores the usage of doctor-rating websites, the determinants of respondents' awareness of the doctor ratings websites, and of their intention to use the sites in the future.

This study brings forward direct evidence suggesting that the awareness and actual usage of doctor-rating websites in the UK remains particularly low. The main finding suggests that the doctor-patient relationship plays a key role in explaining intention to use the websites and that the doctor has both a 'complementary' and 'substitute' role to play with respect to Internet information.

The existence of both 'substitute' and 'complementary' effects between the doctor and the Internet as information channels is not at all conflicting. In fact, they both indicate that the level of concordance achieved during the consultation is likely to define whether or not individuals will seek for further information channels, such as the Internet.

The findings of our study thus contribute also to the wider debate on the inter-relationships between Internet usage and the doctor-patient relationship. ${ }^{7} 25 \quad 26 \quad 32-35$ The argument, sometimes addressed by the previous literature, that information on the Internet can threaten the trust relationship and the balance of roles between doctors and patients, seems a concern which is not supported by our evidence. If any, a potential challenge to the doctor-patient relation can only affect the patients who already feel dissatisfied with the ability of their doctor to listen to them and provide them with enough information regarding their condition, or with the level of their choice for healthcare treatments.

The above, however, can hardly be seen as a serious threat by those who advocate a greater choice by patients. On the contrary, if the latter is indeed a priority in the health policy agenda, online information on 
healthcare providers should be seen as a challenging opportunity to enhance patients' choice in healthcare, and public engagement with health information, especially for the less favoured segments of the population. Indeed, our findings suggest that subjects of non-White background and with lower income are more willing to use online ratings.

Finally, our study highlights that subjects who use doctor-rating websites are unlikely to be representative of the overall patients' pool. In particular, they tend to over-represent opinions from non-White British, medium-to-low-income patients who are not satisfied with their choice of healthcare treatments. Accounting for differences in the users' characteristics is important when interpreting results from doctor-rating sites and when informing interventions that aim at enhancing the public engagement with health information on the Internet, and the representativeness of the users who seek and provide feedback online.

\section{Author affiliations}

${ }^{1}$ LSE Health and Centre for the Study of Incentives in Health, London School of Economics, London, UK

${ }^{2}$ Business School, Imperial College London, London, UK

${ }^{3}$ Department of Health Care Management and Policy, University of Surrey, Guildford, UK

${ }^{4}$ Department of Medicine, Imperial College, London, UK

${ }^{5}$ Department of Medicine, King's College London, London, UK

Contributors MM, MMG and CS contributed to the design of the questionnaire, carried the data analysis, drafted the paper, and amended the paper according to the reviewers suggestions. The remaining authors contributed to the questionnaire design, data collection and drafting of the paper.

Funding This research received no specific grant from any funding agency in the public, commercial or not-for-profit sectors.

Competing interests None.

Provenance and peer review Not commissioned; externally peer reviewed.

Data sharing statement Technical appendix, statistical code and dataset available from the corresponding author at m.miraldo@imperial.ac.uk. Consent for data sharing was not obtained but the presented data are anonymised and risk of identification is low. All authors had full access to all the data in the study and take responsibility for the integrity of the data and the accuracy of the data analysis.

\section{REFERENCES}

1. Department of Health. The NHS Plan: a plan for investment, a plan for reform. Crown 2000. Cm 4818-I.

2. Department of Health. The NHS Improvement Plan: putting people at the heart of public services. London: The Stationery Office, 2004. Cm 6268.

3. Department of Health. Creating a patient-led NHS: Delivering the NHS Improvement Plan. London: The Stationery Office, 2005.

4. Department of Health. High quality care for all: NHS next stage review final report. London: The Stationery Office, 2008. Cm 7432.

5. Dixon A, Robertson R, Appleby J, et al. Patient choice. London: The Kings Fund, 2010. http://www.kingsfund.org.uk/publications/ patient choice.html (accessed 15 Jan 2012)

6. Department of Health. Report of the National Patient Choice Survey, England. Crown 2008.

7. Lagu T, Hannon NS, Rothberg MB, et al. Patients' evaluations of health care providers in the era of social networking: an analysis of physician-rating websites. J Gen Intern Med 2010;25:942-6.
8. Gao GG, McCullough JS, Agarwal R, et al. A changing landscape of physician quality reporting: analysis of patients' online ratings of their physicians over a 5-year period. J Med Internet Res 2012;14: e.38.

9. Appleby J, Alvarez A. Public responses to NHS reform. In British Social Attitudes Survey 22nd Report, London: Sage Publications, 2005.

10. Ybarra M, Suman M. Help seeking behavior and the Internet: a national survey. Int J Med Inform 2006;75:29-41.

11. Cohen J. Statistical power analysis for the behavioural sciences. New York/London: Academic Press, 1969.

12. Office for National statistics. 2001 Census: Key Statistics. 2001 http://neighbourhood.statistics.gov.uk/dissemination/LeadTableView. doa $=3 \& b=276755 \& c=$ hammersmith $\& d=13 \& e=15 \& g=334516 \& \mathrm{i}=1001$ $\times 1003 \times 1004 \& \mathrm{~m}=0 \& \mathrm{r}=1 \& \mathrm{~s}=1273150763921 \&$ enc $=1 \& d s F a m i l y l d=47$ (accessed 10 July 2012)

13. Diaz JA, Griffith RA, Ng JJ, et al. Patients' use of the Internet for medical information. J Gen Intern Med 2002;17:180-5.

14. McMullan M. Patients using the Internet to obtain health information: how this affects the patient-health professional relationship. Patient Educ Couns 2006;63:24.

15. Cotten SR, Gupta SS. Characteristics of online and offline health information seekers and factors that discriminate between them. Soc Sc Med 2004;59:1795-806.

16. Brodie M, Flournoy RE, Altman DE, et al. Health information, the Internet, and the digital divide. Health Affairs 2000;19:255-65.

17. Health on the Net Foundation. HON's fourth survey on the use of the Internet for medical and health purposes, 1999.

18. Fox L, Rainie J, Horrigan A, et al. The online healthcare revolution How the web helps Americans take better care of themselves, Pew Internet and American Life Project, 2000.

19. Baker L, Wagner TH, Singer S, et al. Use of the Internet and e-mail for health care information. JAMA 2003;289:2400-6.

20. Wald HS, Dube CE, Anthony DC. Untangling the Web-The impact of internet use on health care and the physician-patient relationship. Patient Educ Couns 2007:68:218-24.

21. Bertakis KD. The influence of gender on the doctor-patient interaction. Patient Educ Couns 2009;73:356-60.

22. Stevenson FA, Kerr C, Murray E, et al. Information from the Internet and the doctor-patient relationship: the patient perspective-a qualitative study. BMC Fam Pract 2007;8:47.

23. Stavropoulou C. Perceived information needs and non-adherence: evidence from Greek patients with hypertension. Health Expect 2012;15:187-96.

24. McCartney M. Will doctor rating sites improve the quality of care? BMJ 2009:338b:1033

25. Lagu T, Lindenauer PK. Putting the public back in public reporting of health care quality. JAMA 2010;304:1711-12.

26. López A, Detz A, Ratanawongsa N, et al. What patients say about their doctors online: a qualitative content analysis. J Gen Intern Med 2012;27:685-92.

27. Wachter B. The patient will rate you now. 2012. http://community. the-hospitalist.org/2012/03/19/the-patient-will-rate-you-now (accessed 10 Aug 2012)

28. Dutta-Bergman MJ. Health attitudes, health cognitions, and health behaviors among Internet health information seekers: population-based survey. J Med Internet Res 2004;6:e15.

29. Kaiser Family Foundation. Update on consumers' views of patient safety and quality information. Kaiser Family Foundation. 2008 http://www.kff.org/kaiserpolls/posr101508pkg.cfm (accessed 10 July 2012)

30. Greaves F, Pape U, King D, et al. Associations between internet-based patient ratings and conventional surveys of patient experience in the English NHS: an observational study. BMJ Qual Saf 2012;21:600-5.

31. Greaves F, Pape UJ, King D, et al. Associations between Web-based patient ratings and objective measures of hospital quality. Arch Intern Med 2012;172:435-6.

32. Nwosu CR, Cox BM. The impact of the Internet on the doctor-patient relationship. Health Inform J 2000;6:156-61.

33. Broom A. Virtually He@lthy: the impact of internet use on disease experience and the doctor-patient relationship. Qual Health Res 2005; 15:325-45.

34. Gorrindo T. Web searching for information about physicians. JAMA 2008;300:213.

35. Malone M, Mathes L, Dooley J, et al. Health information seeking and its effect on the doctor-patient digital divide. $J$ Telemed Telecare 2005;11(Suppl. 1):S1:25-8. 